\title{
Um Mapeamento Sistemático em Progresso Sobre Internet das Coisas e Educação à Distância
}

\author{
Ricardo Fuzeto ${ }^{1}$, Rosana T. V. Braga ${ }^{1}$ \\ ${ }^{1}$ Instituto de Ciências Matemáticas e Computação - Universidade de São Paulo (USP) \\ São Carlos - SP - Brasil \\ ricardofuz@usp.br, rtvb@icmc.usp.br
}

\begin{abstract}
Resumo. A Internet das Coisas surgiu com a alta disponibilidade de sensores, fundamentando a criação das smart cities. No âmbito da Educação À Distância, ainda são estudadas novas formas de utilizar a IoT, a fim de avançar a educação rumo ao ensino ubíquo, permitindo o aprendizado em qualquer lugar e instante. Este trabalho apresenta um Mapeamento Sistemático preliminar sobre a atual relação entre Internet das Coisas e a Educação À Distância. Os resultados obtidos mostram que a utilização de IoT não é reconhecida na maioria dos casos, apesar de ser amplamente utilizado e já existirem tentativas de criar ambientes de aprendizado ubíquo. Como trabalho futuro, pretende-se completar este mapeamento sistemático.
\end{abstract}

\begin{abstract}
The Internet of Things emerged with the high availability of sensors, being the basis of the creation of smart cities. In the e-learnign domain, it is still being studied new ways of using IoT, to improve education towards ubiquitous learning, allowing learning to take place anywhere and at any time. This work presents an ongoing Systematic mapping about the relationship between IoT and e-learning. Results show that IoT usage is not recognized in most cases, although being widely used and allowing several attempts of creating ubiquitous learning environments. As future work, it is planned to complete this systematic mapping.
\end{abstract}

\section{Introdução}

A computação moderna tem utilizado, com crescente interesse, microprocessadores embarcados em aparelhos móveis. Um dos exemplos mais presentes no dia-a-dia são os celulares, cujo poder computacional cresce rapidamente. Assim, a disponibilidade de sensores capazes de nos informar sobre o ambiente onde estão é cada vez maior, o que implica em uma maior disponibilidade de informação sobre os diversos ambientes de uma cidade. Tais informações possibilitam que os diversos serviços de uma cidade sejam aperfeiçoados de forma inteligente, aumentando a eficiência enquanto minimiza os custos envolvidos. Segundo Zanella et. al.[Zanella et al. 2014], a criação de serviços públicos inteligentes por meio do uso de redes de sensores móveis motiva a criação de smart cities.

Quando se fala de smart cities e a utilização de redes de sensores móveis, começase a falar, também, sobre Internet das Coisas. O livro Inspiring the Internet of Things [Horsager et al. 2016] apresenta, de maneira simples e direta, o que é a Internet das Coisas (IoT) e vários exemplos de como este conceito é aplicado ao redor do mundo, tornando a computação mais ubíqua. O conceito de computação ubíqua é dado por Weiser 
[Weiser 1993] como "um método para melhorar o uso de computadores tornando vários computadores disponíveis em um ambiente físico, porém fazendo-os efetivamente invisíveis ao usuário". Indo mais a fundo, Lyytinen e Yoo [Lyytinen and Yoo 2002] definem que a computação ubíqua se trata, também, de embarcar a tecnologia nos movimentos e interações naturais do ser humano.

No âmbito da Educação À Distância (EAD), alguns pesquisadores afirmam que é possível criar ambientes de aprendizado ubíquo utilizando o paradigma da Internet das Coisas [Möller et al. 2013]. Além disso, Chang et. al. [Chang et al. 2015] reconhecem que o uso de IoT auxilia com alguns problemas comuns de ambientes de ensino monolíticos, como a fragmentação do tempo de aprendizado e materiais de ensino pouco dinâmicos. No entanto, diversos estudos relacionados utilizam o paradigma da Internet das Coisas, porém não reconhecendo isto abertamente.

Visando entender o cenário da EAD, e como a IoT é utilizada como facilitadora do ensino e aprendizado incluindo os cenários em que não é reconhecida, este trabalho apresenta os resultados parciais de um mapeamento sistemático, relacionando EAD e IoT e identificando os estudos relacionados com estes dois conceitos. Como principal objetivo, deseja-se identificar as soluções publicadas que apresentem novas tecnologias para o ensino. Além disso, é desejado avaliar qual o grau de interesse da comunidade científica em desenvolver ambientes de aprendizado ubíquos, permitindo que os alunos possam aprender independentemente do local em que estejam.

Este trabalho é organizado da seguinte forma: na Seção 2 é apresentado o protocolo de mapeamento sistemático utilizado, tendo foco nas informações mais importantes e relevantes para replicação. Na Seção 3 são apresentados os números obtidos após a leitura dos estudos primários, enquanto na Seção 4 esses resultados são discutidos, e algumas possíveis implicações são apresentadas. Por fim, na Seção 5 é feita a conclusão deste trabalho.

\section{Protocolo de Mapeamento}

Revisão Sistemática (Systematic Literature Review - SLR - em inglês) é, segundo Kitchenham et. al. [Kitchenham et al. 2007], "um tipo de estudo secundário que usa uma metodologia bem-definida para identificar, analisar e interpretar todas as evidências relacionadas a uma questão de pesquisa, de forma que seja repetitível e livre de qualquer viés". Os mesmos autores definem um segundo tipo de estudo secundário: o Mapeamento Sistemático (Systematic Mapping - SM). Este tem como objetivo apresentar as evidências de um domínio de estudo em um alto nível de granularidade, agrupando as evidências encontradas em áreas de similaridade e apontando linhas de pesquisa que demandem mais trabalhos e até mesmo SLRs. Petersen et. al. [Petersen et al. 2008] mencionam que a técnica de MS é recomendada quando há a falta de estudos primários relevantes e de alta qualidade. O SM é recomendado, no lugar de uma SLR, quando o tópico é muito abrangente ou quando existem poucas evidências a serem coletadas.

Para realizar este mapeamento, foram seguidas as recomendações de Kitchenham et. al., minimizando qualquer viés durante a seleção dos estudos primários. Nesta seção, são apresentados os detalhes mais importantes do protocolo de mapeamento sistemático, definido para que o processo siga regras rigorosas e que cada estudo seja avaliado segundo os mesmos parâmetros comparativos. 
V Congresso Brasileiro de Informática na Educação (CBIE 2016)

Anais dos Workshops do V Congresso Brasileiro de Informática na Educação (CBIE 2016)

\subsection{Objetivo e Questões de Pesquisa}

O objetivo deste mapeamento sistemático é identificar como as tecnologias relacionadas à internet das coisas são aplicadas na educação à distância. Os estudos de maior interesse devem apresentar formas de utilização destas tecnologias para melhorar a qualidade do ensino, seja tornando o aprendizado mais presente ou aumentando o interesse dos alunos pelos conteúdos ministrados. Com tais objetivos fixados, foram definidas duas questões de pesquisa:

Questão de Pesquisa 1 (RQ1): quais soluções, criadas até hoje, aplicam a internet das coisas na educação à distância?

Questão de Pesquisa 2 (RQ2): dentre os estudos que relacionam internet das coisas com educação à distância, quais enfocam o aprendizado ubíquo?

Nas questões de pesquisa, foi definido que é de suma importância localizar os trabalhos que visam criar ambientes de aprendizado ubíquo. Durante a calibragem das chaves de pesquisa, foi identificada esta terminologia em artigos relevantes, e após pesquisas sobre este tópico específico foi identificada uma relação com o uso de tecnologias de IoT.

\subsection{Termos e Sinônimos de Pesquisa}

A partir das questões de pesquisa apresentadas, foram definidos os termos de pesquisa e alguns dos sinônimos utilizados para cada um deles. Foram utilizadas as tecnologias mais populares de IoT como sinônimos por poucos estudos apresentarem explicitamente a utilização de conceitos de IoT no desenvolvimento das soluções apresentadas. Foi identificado que os pesquisadores interessados em educação raramente reconhecem utilizar conceitos de IoT, apesar de usarem tecnologias popularmente relacionadas.

\begin{tabular}{|l|l|}
\hline Internet of Things & $\begin{array}{c}\text { Internet das Coisas, IoT, RFID, WSN, } \\
\text { Wireless Sensor Network, sensor }\end{array}$ \\
\hline Educação à distância & $\begin{array}{c}\text { EAD, E-learning, ubiquitous learning, } \\
\text { u-learning }\end{array}$ \\
\hline
\end{tabular}

Tabela 1. Termos de busca e sinônimos

As bases de dados consultadas, utilizando os termos e sinônimos de busca apresentados na Tabela 1, foram: ACM Digital Library, IEEE Xplore, Scopus, SpringerLink e ScienceDirect. O Google Scholar também foi consultado, porém apenas como uma base de dados auxiliar, a fim de verificar se a chave de busca estava adequada segundo os números obtidos nas outras bases.

\subsection{Critérios de Inclusão e Exclusão}

Critérios de inclusão e exclusão foram definidos para auxiliar na condução do mapeamento, facilitando a classificação de relevância dos estudos. Durante a coleta de estudos, identificou-se que diversos estudos relevantes não reconhecem explicitamente o uso de conceitos de IoT. Desta forma, foi definido um critério de inclusão, o qual recomenda que estudos mencionando explicitamente o uso de IoT sejam aceitos como estudos primários. 
V Congresso Brasileiro de Informática na Educação (CBIE 2016)

Anais dos Workshops do V Congresso Brasileiro de Informática na Educação (CBIE 2016)

Assim, estudos que falam explicitamente sobre IoT terão maior possibilidade de serem aceitos. Além disso, os estudos que não apresentam esta discussão não serão excluídos por este motivo.

Os critérios de exclusão definidos somam 4 critérios distintos, que são:

Critério de Exclusão 1 (EC1): todos os estudos que não falam sobre educação à distância e sobre Internet das Coisas, mesmo implicitamente, serão excluídos.

Critério de Exclusão 2 (EC2): caso haja um conjunto de estudos muito próximos, ou com contribuições muito semelhantes, apenas um será aceito, excluindo todos os outros. Este critério se estende a estudos duplicados.

Critério de Exclusão 3 (EC3): todos os resultados, obtidos nas bases de dados, que não forem estudos, como sumários de conferências e capas de journals, não serão excluídos. Porém, ao invés de serem usados como estudos primários, serão considerados como fontes de consulta para identificar futuros tópicos de interesse e tendências de pesquisa.

Critério de Exclusão 4 (EC4): todos os estudos que não puderem ser lidos serão, infelizmente, excluídos.

\section{Resultados Obtidos}

No momento em que este artigo foi escrito, apenas os resultados das bases de dados ACM Digital Library e IEEE Xplore haviam passado por todos os ciclos de execução deste mapeamento sistemático. Portanto, é de suma importância lembrar que os resultados obtidos são preliminares. Por isso, considera-se, neste momento, apenas os 276 resultados obtidos destas bases de dados.

Dos 276 resultados obtidos na primeira consulta, 128 foram selecionados para 0 primeiro ciclo de execução. Para realizar a leitura dos estudos, foi selecionada a abordagem em três passos, segundo Keshav [Keshav 2007]. Ao final dos três ciclos de leitura dos estudos, 45 foram aceitos como estudos primários. Nos Gráficos 1 e 2 pode-se ver a distribuição destes estudos primários por ano e país de origem, respectivamente.

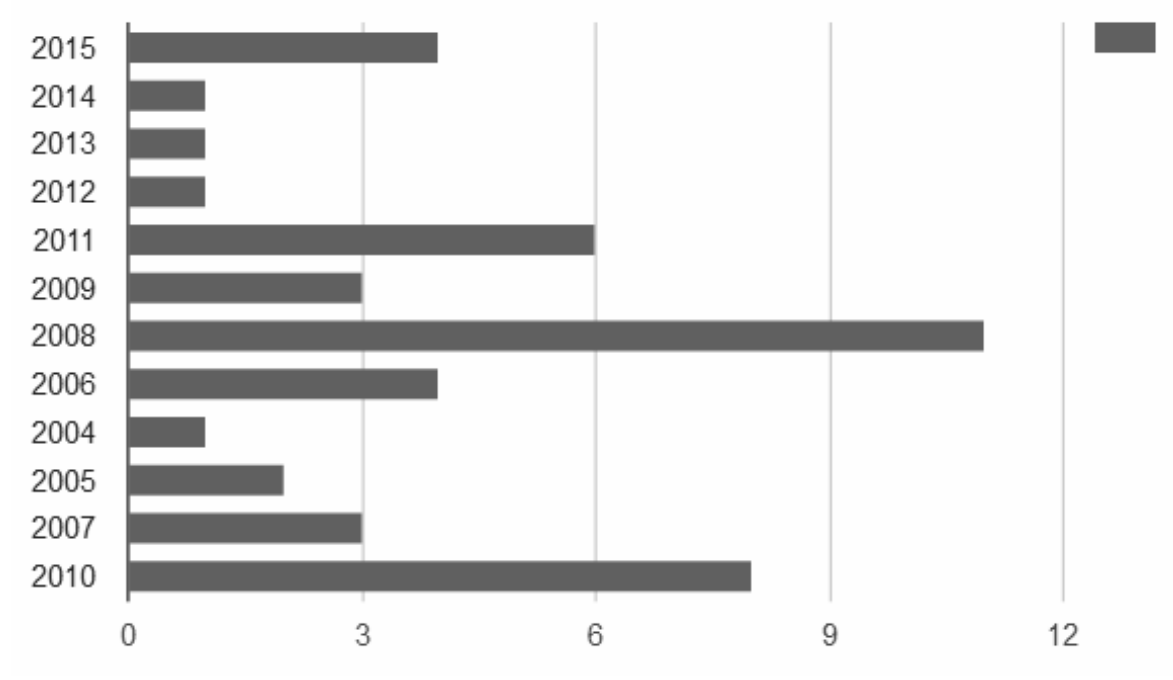

Gráfico 1. Publicações por ano 
V Congresso Brasileiro de Informática na Educação (CBIE 2016)

Anais dos Workshops do V Congresso Brasileiro de Informática na Educação (CBIE 2016)

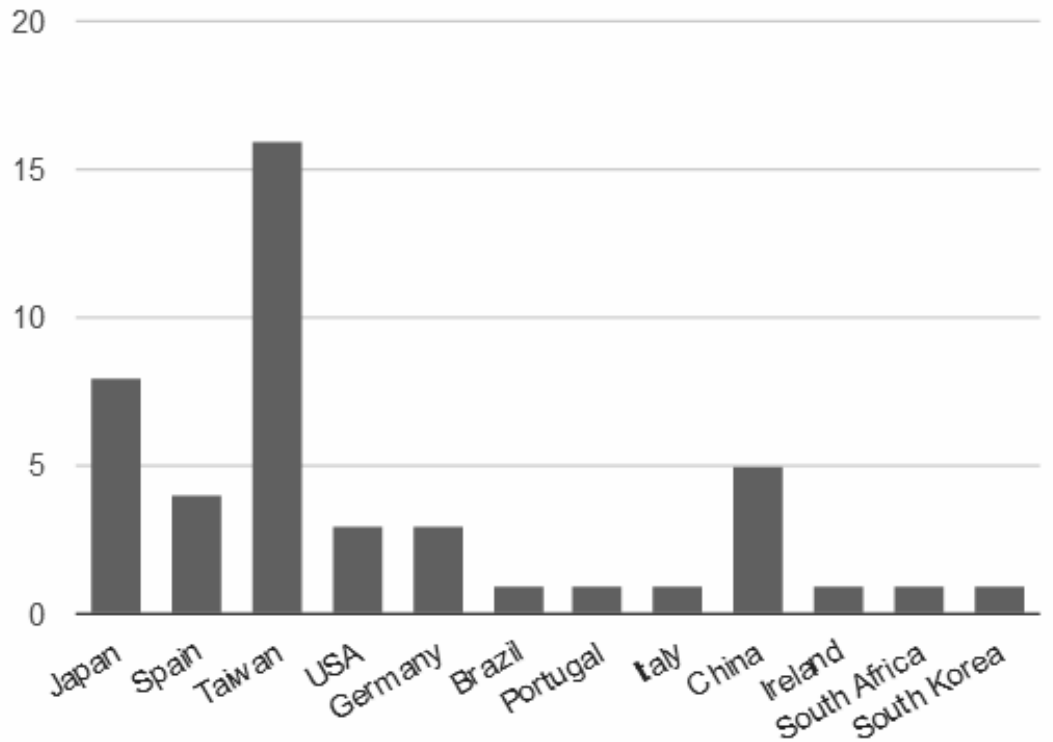

Gráfico 2. Publicações por país

Cada estudo poderia apresentar a solução aplicada em um domínio de ensino, a fim de verificar em quais aspectos do processo de aprendizagem suas soluções seriam efetivas, além de obter dados experimentais de sua aplicação. Assim, foi verificado quais estudos apresentam alguma aplicação, e em quais domínios especificamente eles aplicam suas ideias. Os números obtidos são apresentados no Gráfico 3.

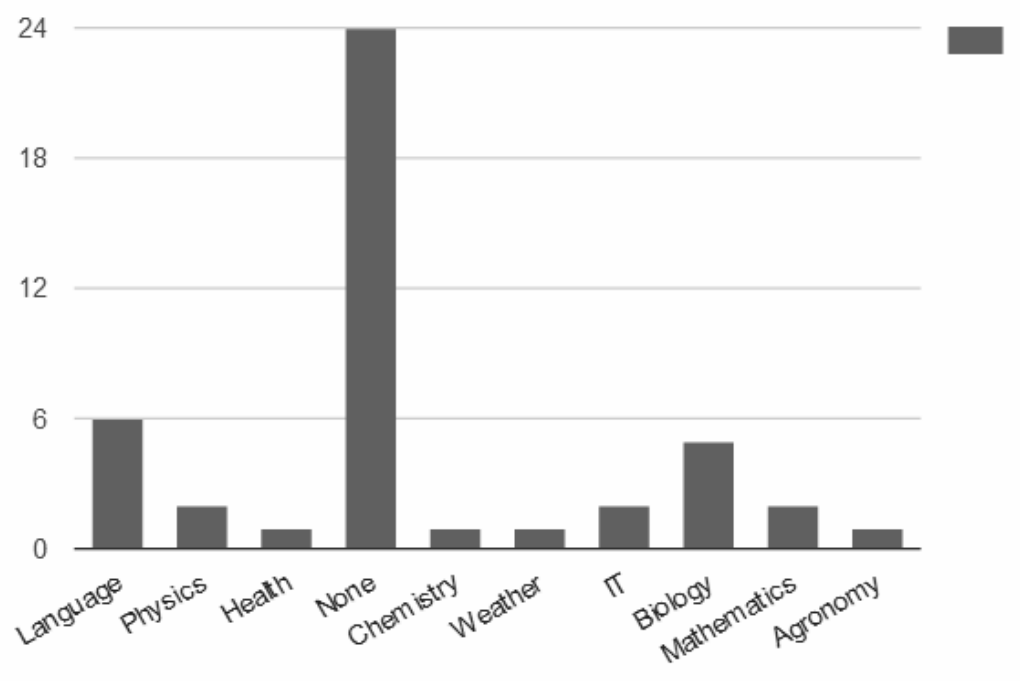

Gráfico 3. Domínios de ensino utilizados

Por fim, um dos últimos dados coletados neste mapeamento sistemático se refere ao tipo de ambiente desenvolvido e/ou considerado em cada estudo. É fundamental mencionar que os ambientes de interesse são ambientes que possibilitem o ensino na modalidade EAD. Por isso, classifica-se os ambientes obtidos em três tipos diferentes: os ambientes EAD monolíticos, mais comumente utilizados, nomeados e-learning segundo sua nomenclatura em inglês; ambientes $m$-learning, que aprimoram ambientes de EAD 
V Congresso Brasileiro de Informática na Educação (CBIE 2016)

Anais dos Workshops do V Congresso Brasileiro de Informática na Educação (CBIE 2016)

monolíticos com a incorporação de tecnologias que permitam o aprendizado e/ou ensino utilizando dispositivos móveis, facilitando o processo de alguma forma; e ambientes $u$ learning, que tornam o aprendizado ubíquo e, portanto, aproximando o processo de ensino e aprendizado da possibilidade de ocorrer em qualquer ambiente. O número de estudos por tipo de ambiente é mostrado no Gráfico 4. É importante citar, também, que alguns estudos não apresentam a utilização de um ambiente de ensino.

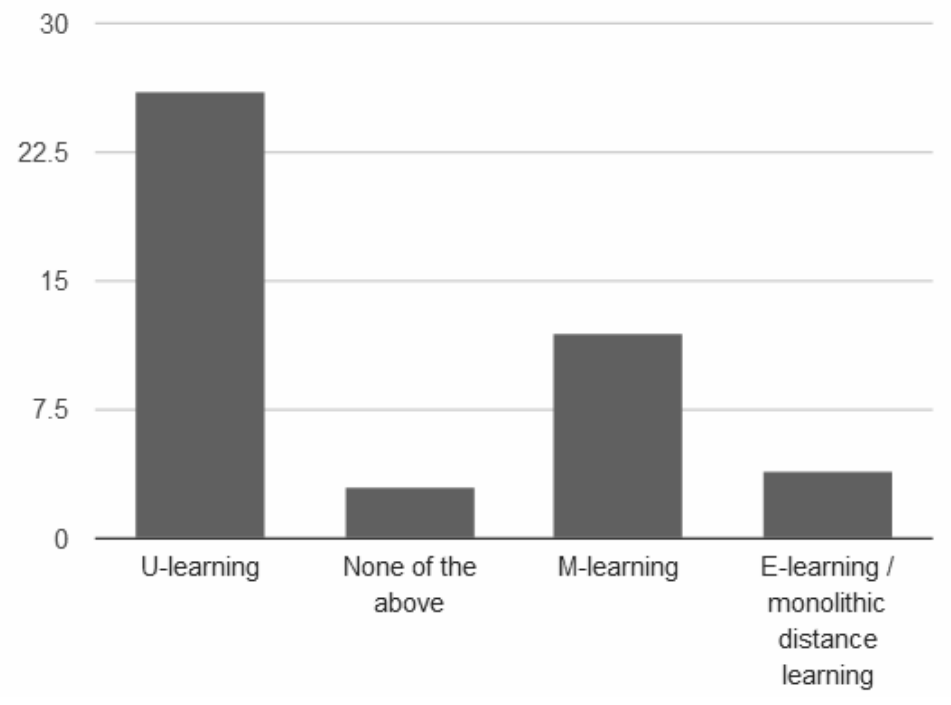

Gráfico 4. Tipos de ambientes de Ensino À Distância

\section{Discussão dos Resultados}

Conforme pode-se observar no Gráfico 1, há um grande volume de publicações entre os anos de 2008 e 2011, o que demonstra um recente interesse neste tema. Pode-se observar isto, também, pelo número de publicações no ano de 2015. Das 11 publicações de 2008, 8 focam ambientes de aprendizado ubíquo, o que demonstra que o interesse por este tipo de ambiente não é tão recente. Indo além, em apenas 2 anos não foram encontrados estudos sobre ambientes de aprendizado ubíquo, em 2009 e 2014.

Os continentes que mais publicam sobre o tema (Internet das Coisas e Educação À Distância) são a Ásia e Europa, tendo publicado 30 e 10 estudos, respectivamente. É notável o volume de estudos publicados somente em Taiwan, totalizando 16 estudos, o que significa cerca de $35 \%$ dos estudos primários selecionados. Com isto, pode-se evidenciar que a região da Eurásia apresenta um maior engajamento em pesquisar tecnologias de EAD. É possível notar que alguns continentes, como Américas do Norte e Sul e África, figuram no gráfico com alguns estudos publicados, o que faz da América Central e da Oceania os únicos continentes a não terem estudos publicados selecionados neste trabalho. Apesar de uma única contribuição, o Brasil aparece neste cenário com um estudo utilizando um ambiente de ensino ubíquo.

Outra discussão que deve ser feita é sobre os tipos de estudos publicados. Conforme os resultados obtidos, dos 45 estudos primários, 26 são classificados como experimentais, enquanto os 19 restantes são teóricos. Isto representa uma preocupação, por parte da comunidade científica, em encontrar novas soluções e avaliar seu impacto com 
V Congresso Brasileiro de Informática na Educação (CBIE 2016)

Anais dos Workshops do V Congresso Brasileiro de Informática na Educação (CBIE 2016)

experimentos. No entanto, o volume semelhante de estudos teóricos mostra que soluções ainda não avaliadas de forma prática também são aceitas.

As tecnologias relacionadas a IoT utilizadas nos estudos podem ser relacionadas com os tipos de recursos educacionais utilizados. Segundo os dados apresentados no Gráfico 5, cerca de 50\% dos estudos não usam um dos recursos elicitados no formulário de coleta de dados, ou sequer utilizam algum recurso educacional. Isto indica que há um grande volume de estudos relevantes que reúnem informações sobre o tema, ou propõem um avanço tecnológico mas sem a condução de experimentos. Também é possível ver aproximadamente $37 \%$ dos estudos utilizando ambientes orientados ao ensino e aprendizado. Quanto às tecnologias de IoT, de acordo com o Gráfico 6, a maioria dos estudos utilizam etiquetas RFID, redes de sensores sem-fio ou a combinação das duas tecnologias. Também há um grande volume de uso de aparelhos móveis pessoais, como PDAs e celulares smartphone. Isto pode representar uma tendência em desenvolver sistemas de ensino e aprendizado utilizando estas abordagens, o que pode ocasionar, por exemplo, deficiências nas tecnologias relacionadas a sistemas na nuvem, tendo em vista que apenas 3 estudos utilizam este tipo de tecnologia.

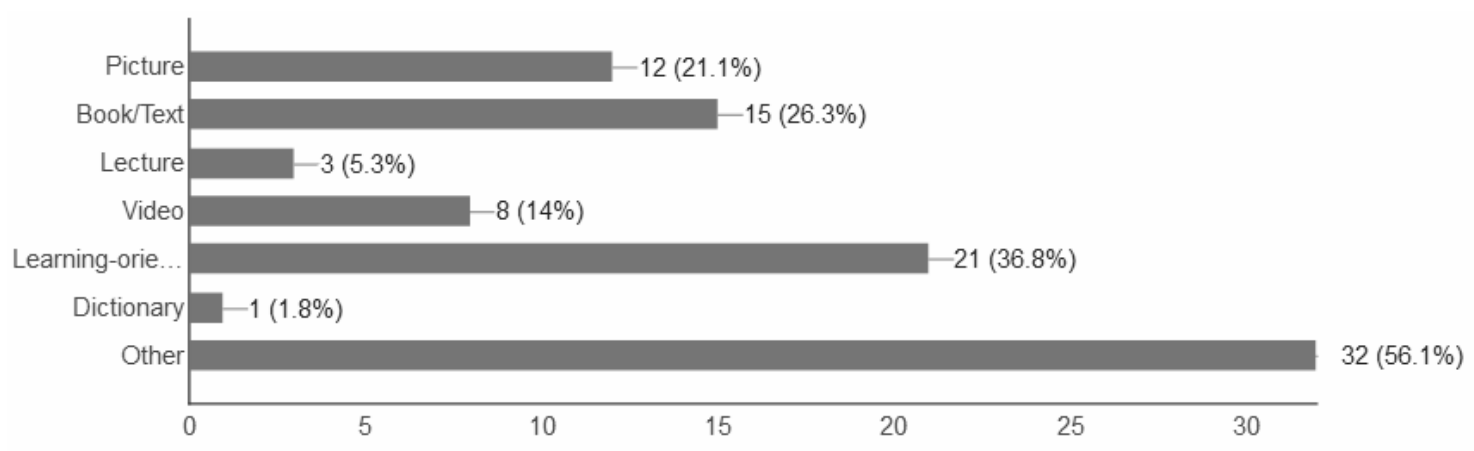

Gráfico 5. Tipos de recursos educacionais utilizados

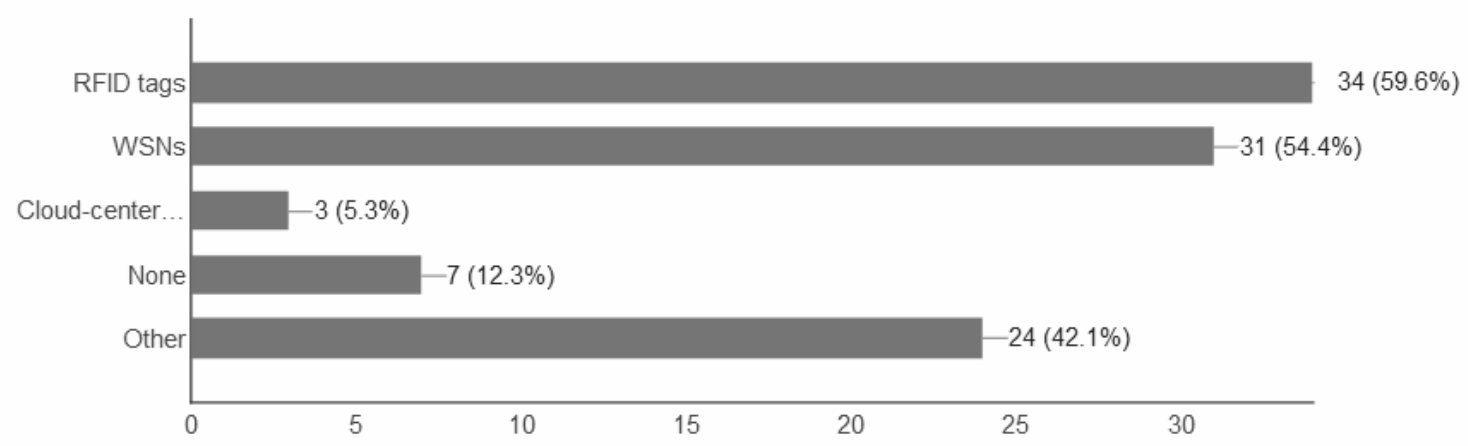

Gráfico 6. Tecnologias de loT utilizadas

\subsection{Tecnologias e Abordagens Propostas}

As discussões feitas nesta seção resumem, dentre os estudos primários selecionados, as tecnologias e abordagens publicadas consideradas mais inovadoras. Dois estudos apresentam o uso de um middleware: um software de apoio, que oferece interfaces entre sistemas operando mais próximos do sistema operacional, e aplicações 
que se deseja desenvolver no domínio de suporte do middleware. O trabalho de [Boyinbode and Bagula 2011] apresenta um middleware para sistemas educacionais ubíquos, e que também oferece suporte à pessoas com deficiências. Já o trabalho de [Martin et al. 2009] reconhece diversos problemas da utilização de tecnologias de IoT, como sensores móveis e a mobilidade dos usuários, e aplica soluções orientadas à mitigação desses problemas em um middleware para sistemas m-learning.

Alguns dos estudos selecionados investigam o potencial de conteúdos educacionais criados pelos próprios usuários interessados em aprender. $\mathrm{O}$ trabalho de [Mouri et al. 2015] propõe um sistema em que o usuário pode registrar situações de aprendizado, e recebe sugestões de novos lugares onde há chances de acumular novos conhecimentos. Este estudo se destaca por exigir que o usuário tenha apenas um smartphone, sem precisar que novos equipamentos estejam presentes no ambiente. $\mathrm{O}$ trabalho de [Ogata 2008] compila uma série de aplicações, feitas no mesmo instituto pelo mesmo grupo de pesquisadores, que auxiliam os usuários a aprenderem a língua japonesa e as expressões corporais corretas, segundo os costumes japoneses. Os sistemas JAMIOLAS e JAPELAS sugerem as expressões corretas segundo as informações inseridas pelo usuário, enquanto o TANGO permite que o usuário aprenda os nomes e vocábulos de objetos próximos por meio de etiquetas RFID próximas, marcando os objetos que possam ensinar algo. Além destes três aplicativos, o LORAMS, também criado pelo grupo de pesquisadores, permite que os usuários gravem vídeos sobre algum aprendizado adquirido, e enviam este artefato ao sistema via smartphone.

Möller et. al. [Möller et al. 2013] discutem o conceito de $u$-learning mais a fundo. Os autores apresentam a definição de lifelong learning como a habilidade de um sistema em manter o interesse de um usuário pelo aprendizado ao longo de toda a sua vida. Baseando-se nesse conceito, é apresentada uma relação de convergência entre diferentes abordagens de ensino e tecnologias utilizáveis. Os autores reconhecem que os dispositivos utilizados em um ambiente de ensino ubíquo devem possuir três características: rastreabilidade, identificação e conexão mútua. Os autores também apresentam características fundamentais, para este tipo de sistema, a fim de garantir a máxima qualidade. Por fim, é apresentado um estudo de caso baseado em um curso de modelagem e simulação, em que os autores propõem um espaço de aprendizado ubíquo relacionado.

O trabalho de Gad [Gad 2011] apresenta um exemplo de sistema de aprendizado ubíquo específico. O autor criou um sistema que coleta os sinais de um sensor de Eletroencefalograma (EEG), usado por um usuário voluntário, e envia os resultados via smartphone para um servidor. Os dados armazenados podem ser acessados por outro dispositivo, para que o aluno possa analisar os sinais, e avaliar a condição do usuário. Este sistema visa o treinamento de novos médicos, para a leitura de sinais de atividade cerebral. No entanto, o autor reconhece que os dados coletados são massivos para um dispositivo portátil, por possuírem pouca memória disponível. Por isso, a utilização de algoritmos baseados em MapReduce é capaz de reduzir a quantidade de dados, tornando menor, dessa forma, a massa de dados utilizada e o poder computacional necessário. Ainda está em aberto o problema do consumo de bateria e a execução de experimentos com sensores reais.

Por fim, o trabalho de [Liu and Chu 2008] apresenta como é possível criar um ambiente de aprendizado móvel utilizando técnicas de realidade aumentada (Augmented 
Reality - AR). O sistema proposto, HELLO, utiliza aparelhos óticos de realidade aumentada como elemento-chave do sistema, uma vez que são necessários para que a AR do sistema funcione. Por meio destes aparelhos, o usuário pode enxergar tutores virtuais nos diferentes ambientes onde o sistema funciona, além de informações oferecidas para melhorar o aprendizado. O usuário também pode executar operações no sistema utilizando um dispositivo móvel, tendo sido usado um PDA (Personal Digital Assistant) nos experimentos feitos. Segundo os autores, os resultados mostram que os usuários tiveram um melhor aprendizado ao usar o sistema, e que gostariam de usá-lo em outras áreas de conhecimento. No entanto, os autores reconhecem a pouca portabilidade dos dispositivos óticos como uma grande barreira para a utilização deste sistema no cotidiano dos alunos.

\section{Conclusões}

O mapeamento sistemático realizado, ainda em progresso, mostrou uma seleção de estudos que relacionam a internet das coisas com a educação à distância. Foi identificado que diversos autores não reconhecem estarem utilizando IoT explicitamente nos estudos publicados, e que, por isso, este trabalho se mostra importante para a comunidade científica envolvida para que este conceito seja formalmente apresentado, permitindo, assim, uma melhor comunicação entre especialistas do domínio.

Futuramente, planeja-se a conclusão deste mapeamento sistemático, o que deverá fornecer a comprovação dos resultados aqui apresentados, apesar da pequena massa de estudos primários obtidos até o momento. A conclusão deste SM envolve a análise e interpretação dos estudos obtidos das bases de dados ScienceDirect, Scopus e SpringerLink. As lacunas de pesquisa identificadas por meio deste mapeamento poderão originar diversos trabalhos de pesquisa que auxiliarão desenvolvedores de aplicações para educação a distância no uso de tecnologias IoT. Em particular, os autores estão interessados em como aplicar os conceitos de engenharia de software no desenvolvimento de aplicações que usam tecnologias IoT.

\section{Agradecimentos}

Agradecemos ao Conselho Nacional de Desenvolvimento Científico e Tecnológico (CNPq) e à Universidade de São Paulo (USP) por permitirem que este trabalho seja realizado.

\section{References}

Boyinbode, O. and Bagula, A. (2011). An adaptive and personalized ubiquitous learning middleware support for handicapped learners. In Proceedings of the 2011 Eighth International Conference on Information Technology: New Generations (ITNG), pages 632-637. IEEE.

Chang, C. W., Lin, P., Tseng, C. W., Kong, Y. K., Lien, W. C., Wu, M. C., and Wu, C. Y. (2015). Poster: Design and implementation of iot-enable mobile e-learning platform for medical education. In Proceedings of the 16th ACM International Symposium on Mobile Ad Hoc Networking and Computing, pages 385-386. ACM New York.

Gad, S. H. (2011). Cloud computing and mapreduce for reliability and scalability of ubiquitous learning systems. In Proceedings of the compilation of the co-located workshops 
V Congresso Brasileiro de Informática na Educação (CBIE 2016)

Anais dos Workshops do V Congresso Brasileiro de Informática na Educação (CBIE 2016)

on DSM'11, TMC'11, AGERE! 2011, AOOPES'11, NEAT'11, \& VMIL'11, pages 273278. ACM New York.

Horsager, H., Raun, T., Skotting, M., Presser, M., Andersen, S., Larsen, M. K., and Contributors (2016). Inspiring the Internet of Things. available at https://iotcomicbook.org/.

Keshav, S. (2007). How to read a paper. In ACM SIGCOMM Computer Communication Review, volume 37(3), pages 83-84.

Kitchenham, B., Charters, S., Budgen, D., Brereton, P., Turner, M., Linkman, S., Jørgensen, M., Mendes, E., and Visaggio, G. (2007). Guidelines for performing systematic literature reviews in software engineering. Technical report, Keele University, UK.

Liu, T. and Chu, Y. (2008). Handheld augmented reality supported immersive ubiquitous learning system. In Proceedings of the 2008 IEEE International Conference on Systems, Man and Cybernetics, pages 2454-2458. IEEE.

Lyytinen, K. and Yoo, Y. (2002). Ubiquitous computing. In Communications of the ACM, volume 45(12), pages 63-65. ACM New York.

Martin, S., Gil, R., Cristobal, E., Díaz, G., Castro, M., Peire, J., Milev, M., and Mileva, N. (2009). Middleware for the development of context-aware applications inside mlearning: Connecting e-learning to the mobile world. In Proceedings of the Fourth International Multi-Conference on Computing in the Global Information Technology (ICCGI) '09, pages 217-222. IEEE.

Möller, D. P. F., Haas, R., and Vakilzadian, H. (2013). Ubiquitous learning: Teaching modeling and simulation with technology. In Proceedings of the 2013 Grand Challenges on Modeling and Simulation Conference(24). Society for Modeling \& Simulation International.

Mouri, K., Ogata, H., and Uosaki, N. (2015). Ubiquitous learning analytics in the context of real-world language learning. In Proceedings of the Fifth International Conference on Learning Analytics And Knowledge, pages 378-382. ACM New York.

Ogata, H. (2008). Computer supported ubiquitous learning: Augmenting learning experiences in the real world. In Proceedings of the Fifth IEEE International Conference on Wireless, Mobile, and Ubiquitous Technology in Education (WMUTE) 2008, pages 3-10. IEEE.

Petersen, K., Feldt, R., Mujtaba, S., and Mattson, M. (2008). Systematic mapping studies in software engineering. In Proceedings of the 12th international conference on evaluation and assessment in software engineering, volume 17(1).

Weiser, M. (1993). Some computer science issues in ubiquitous computing. In Communications of the ACM, volume 36, pages 75-84. ACM New York.

Zanella, A., Bui, N., and Castellani, A. (2014). Internet of things for smart cities. In IEEE Internet of Things Journal, pages 22-32. IEEE. 